\title{
ST VARIABILITY ASSESSMENT BASED ON COMPLEXITY FACTOR USING INDEPENDENT COMPONENT ANALYSIS, HILBERT TRANSFORM AND REGRESSION ANALYSIS
}

\author{
S. Thulasi Prasad ${ }^{1}$, S. Varadarajan ${ }^{2}$ \\ ${ }^{1}$ Assoc. Professor, Dept. of ECE, Sree Vidyanikethan Engineering College, Tirupati, India,stprasad123@yahoo.co.in \\ ${ }^{2}$ Professor, Dept. of ECE, S. V. University College of Engineering, Tirupati, India, varadasouri@gmail.com
}

\begin{abstract}
In recent days the computerized ECG has become the most effective and convenient diagnostic tool to identify cardiac diseases such as Myocardial Ischemia (MI). Among the Cardio vascular diseases (CVDs) the Myocardial Ischemia (MI) is one of the leading causes of heart attacks. The Myocardial Ischemia (MI) occurs due to the difficulties in the flow of the electrical impulses from SA node to bundle branches because of the abnormalities in the conduction system. Normally the ECG is used as a main diagnostic tool to identify the cardiac diseases. In order to obtain accurate information from ECG it is necessary to remove all the artifacts and extract the pure ECG from noise background. In this paper the removal of the artifacts is achieved with linear filtering and the extraction of the clean ECG signal is performed using Independent Component Analysis (ICA). After preprocessing and ECG extraction, the QRS complex of each beat is detected by using Hilbert Transform and simple threshold detection algorithm. Next the Instantaneous Heart Rate (IHR) from RR interval and Complexity Factor (CF) from time series ST segment are computed for each beat to form desired feature sets. Later a linear regression model is designed using Instantaneous heart rate (IHR) and ST segment Complexity Factors (STCFs) based on Linear Regression analysis. The proposed ICA-STCFR model is used to identify the ischemic beats from the test feature sets of ECG signal to assess the ST-Segment Variability (STV). The ECG data sets obtained from a local hospital were used to design and test the model. The evaluation parameters, Ischemic Intensity Factor (IIF), Ischemic Activity Factors (IAF) and Peak to Average Value (PAV) were used to evaluate the proposed method and compared with Wavelet Transform based method. The proposed ICA-STCFR was found to be yielding better results than WT-ST method.
\end{abstract}

Key Words: Myocardial Ischemia, ICA, HT, QRS Complex, RR interval, ST segments, IHR, STCF, Scatter-plot

\section{INTRODUCTION}

In present situation, the bioengineering is becoming a vital course in engineering courses as it can be used to solve a number of difficulties which are being faced by the researchers and doctors in diagnosing ailments. The Digital Signal Processing (DSP) in bioengineering deals with the analysis of signals such as filtering, smoothing, digitization, feature extraction, prediction, in either discrete or continuous time, to dig out useful information from those signals etc.

The Electro Cardio Gram (ECG) is the deflection produced by the electric charge picked up by a set electrodes placed on the chest near the heart and limbs of the human body. This gives a waveform representation of the electrical activity of human heart. The deviations in the normal electrical patterns indicate various cardiac disorders. During the cardiac cycle of human heart the bioelectrical action potentials are transmitted through the various parts of the heart to perform contractions and relaxations of cardiac muscles in a rhythmic way. These electrical potentials from surface of the chest near the heart are recorded as an ECG [1], [2]. It is used as a basic investigative and major diagnostic tool by the cardiologists in cardiology to detect and analyze the Heart Rate Variability, Auricular and Ventricular Hypertrophy, Myocardial Ischemia and Infarction (heart attack), Arrhythmias and Pericarditis [3], [4]. These imperfections are reflected by the variation of the intervals and magnitudes of the $\mathrm{P}$ wave, QRS complex, $\mathrm{T}$ wave, PR segment, QT interval, ST interval, PR segment, and ST segment of the ECG signal. The analysis of these can help the doctors to diagnose human heart diseases accurately. Fig-1 shows the ECG wave containing plenty of information pertaining to the patient's heart. The ST segment is the portion of the ECG wave that lies between the J-point and the K-point [5]. This is normally flat in a healthy subject. In case of defective subject ST segment is not always flat instead it is elevated or depressed.

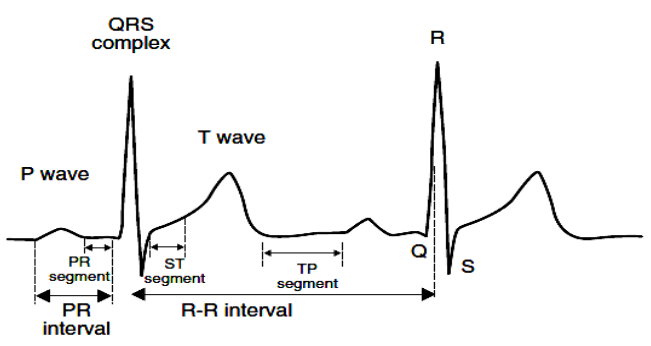

Fig-1: ECG with characteristic features 
The measurement of this deviation of ST segment by using normally existing detection techniques is not always easy and accurate as the non Gaussian noise is mixed with ECG. The most commonly used method in the real world applications are Pan-Tompkins QRS detection algorithm and its modification introduced by Hamilton and Tompkins [6], [7]. Pan-Tompkins and its modified HamiltonTompkins algorithms are based on patient specific detection threshold. These algorithms are well suitable to enhance only the positions of QRS complexes and not the locations of onset and off set of ST segment. This difficulty is overcome by the Hilbert Transform and Independent Component Analysis (ICA).

\section{LITERATURE REVIEW}

Shook et al (1989) performed analysis on HR and ST variability to ECG beats for detecting ischemia. In this work the maximum and mean ST deviation was measured by taking deviation of ST segment as the deviation of ECG signal from at a point that lies $80 \mathrm{~ms}$ after $\mathbf{J}$ from the isoelectric line and also the average ST slope computed for every $30 \mathrm{~s}$ of the ECG signal. Badilini et al (1992) performed analysis on the ST segment in the frequency domain and created a system to detect ischemia. Taddei et al (1995) employed a geometric algorithm for estimation of the ST segment from two lead ECG recordings by developing a rule-based system. In this method it is assumed that an ischemic episode contains only ischemic beats and the information only from the ST segment was used. In their analysis it was observed that the ischemic beats are rich in lower frequencies than the normal ones.

Andreao RV et al (2004) proposed an ECG analysis system based on Markov Model for beat segmentation and ischemia detection. Ali Zifan et al. (2008) developed an automated approach for annotating ECG's. This approach involves segmentation of ECG using Adaptive piecewise constant approximation (APCA) and Piecewise derivative dynamic time warping (PDDTW). Single lead segmentation method was used to segment ECG to reduce annotation time of ECG's greatly instead of manual annotation.

Roshan Joy Martis et al (2009) used Gaussian mixture model (GMM) to suggest an automatic classifier for detecting cardiac irregularities from ECG. In this suggestion GMM has been used for classification after estimating linear prediction (LP) model, calculating residual error signal, and registration of linearly independent ECG features using Principle Component Analysis (PCA). Mohamed Ben Messaoud et al. (2009) designed an algorithm to detect with Atrial Fibrillation (AF) and ventricular tachycardia based on $\mathrm{P}$ wave characteristics. This method involves recognition of fiducial point such as $\mathrm{R}$ peaks by means of the Pantompkins algorithm, segmentation of the $\mathrm{P}$ waves and utilization of the correlation and regression to compute the similarity factors between a test $\mathrm{P}$ wave segments and the reference segments.

Mishra (2010) developed a classifier to classify ECG in to arrhythmia and normal ECG using a local fractal dimension based on nearest neighbor classification. Dusit Thanapatay (2010) proposed a method using Principle Components Analysis (PCA) and Support Vector Machines (SVM) classifier to explore abnormalities from ECG in heart functioning. Gu-Young Jeong (2010) proposed an algorithm based on the polynomial approximation to identify the ST level changes and then to classify them according to the ST shape. In this research paper the ST shape is used as an important diagnostic parameter for detecting myocardial ischemia.

M. K. Islam et al. (2012) discussed in their published paper the various digital signal processing techniques to analyze ECG signals by means of MATLAB tools. They focused on the study of simulation, acquisition, filtering and feature extraction of real time ECG data. T Yingthawornsuk et al. (2012) presented a paper on Classification of Cardiac Arrhythmia based on SVM. In this paper Support Vector Machine (SVM) classifier utilized to separate multi - class arrhythmia dataset. Md. Ashfanoor Kabir et al. (2012) carried out denoising of ECG signal using Empirical Mode Decomposition (EMD) and Discrete Wavelet Transform (DWT). The ECG signal was denoised in EMD analysis by disposing the number of intrinsic mode functions (IMFs), and denoised in wavelet transform domain by setting the appropriate threshold value to decomposition levels.

Chetan. G et al (2014) proposed an adaptable PLI canceller utilizing Least Mean Square (LMS) algorithm to remove $50 / 60 \mathrm{~Hz}$ powerline interference from electrocardiogram (ECG).

\section{METHODOLOGY}

In this section the proposed method has been described with help of the block diagram as shown in the Fig-2.

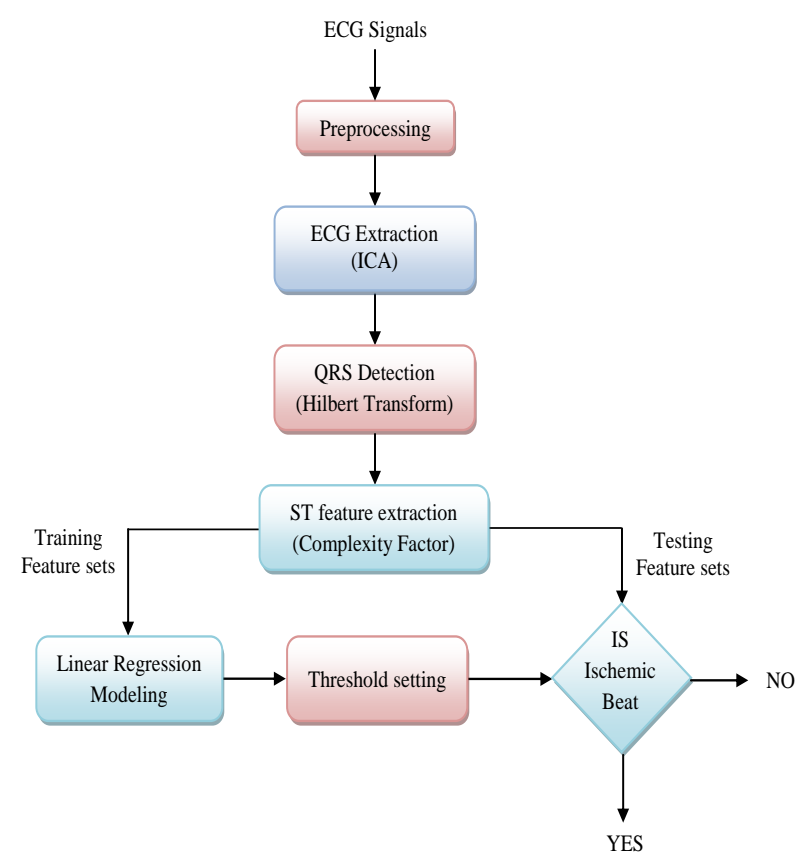

Fig-2: Block diagram of ICA-STCFR modeling for ST segment Variability Analysis 
This method consists of preprocessing, ECG signal extraction, QRS detection, STCF feature extraction, STCFR modeling and beat classification. This method requires the ST segment Complexity Factors (STCF) and Instantaneous Heart Rate (IHR) of each heart cycle in order to form feature sets for the purpose of regression modeling between STCFs and IHRs. Finally the Ischemic beats of the ECG signals are detected using STCFR model.

\subsection{Preprocessing}

The noise and artifacts due to baselinewanders, power line interference and motion artifacts are removed in preprocessing by performing linear filtering on corrupted ECG [8]. The preprocessing involves three steps: 1. High frequency noise motion and artifacts removal; 2. Baseline wanders removal and 3. Powerline interference removal.

The bandpass digital filter with the passband of $0.05 \mathrm{~Hz}$ to $40 \mathrm{~Hz}$ is used to remove high-frequency noise and the baselinewanders from ECG signal [9]. There are several techniques to remove powerline interference but here in this paper a notch filter, designed for cutoff frequency of $50 \mathrm{~Hz}$, is employed for removing powerline interference noise from ECG signal [10].

\subsection{Independent Component Analysis}

In this paper Independent Component Analysis (ICA) is used to extract the ECG signal from 12 lead ECG by reducing multidimensionality. It is one of the Blind Source Separation Techniques available for extracting biosignals from the noise background [11]. ICA is used to identify and separate mixtures of sources with little prior information. ICA is distinguished from other methods as it looks for components that are both statistically independent and non Gaussian [12]. The ECG is considered as a mixture of signals from nodes presented in the heart, various artifacts and noise [13], [14]. Based on this consideration, ICA is applied on preprocessed ECG signal to separate the ECG from other noise components effectively. Once the ECG is extracted from noise and artifacts it is easy to perform accurate estimation of fiducial points of ECG.

The principle of basic ICA structure is described in the Fig-3. The vectors $\boldsymbol{X}$ and $\boldsymbol{S}$ represent the observed and source signals respectively. A is an unknown matrix of size, $\boldsymbol{n} \boldsymbol{x} \boldsymbol{n}$, and called the mixing matrix. $\boldsymbol{X}$ and $\boldsymbol{S}$ get the size, $\boldsymbol{n} \boldsymbol{x} \boldsymbol{m}$, representing ' $\boldsymbol{n}$ ' number of sources and ' $\mathrm{m}$ ' number of samples in each source.

$$
X=A S
$$

Incidentally, the justification for the description of this signal processing technique as blind is that we have no information on the mixing matrix, or even on the sources.

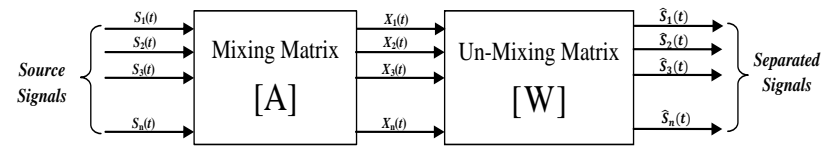

Fig-3: Block diagram of Independent Component Analysis
The objective is to recover the original signals, $\mathrm{S}$ from only the observed vector $\boldsymbol{X}$. Denoting the output vector by $\boldsymbol{V}$, the aim of ICA algorithms is to find a matrix $U$ to undo the mixing effect. That is, the output will be given by

$$
V=U X
$$

Where, $\mathrm{V}$ is an estimate of the sources. The sources can be exactly recovered if $\boldsymbol{U}$ is the inverse of $\boldsymbol{A}$ after a permutation and scale change. The required ECG can be reconstructed by using (3), where ' $\boldsymbol{V}$ ' is the matrix of derived Independent Components (ICs) with the row representing the noise or artifacts set to zero.

$$
X=U^{-1} V
$$

The ICA methods try to estimate components that would be as independent as possible and their linear combination should be the original data. Normally the estimation of components is done either by iterative algorithm, which maximizes function of independence, or by a non-iterative algorithm, which is based on joint diagonalisation of correlation matrices [15]. ICA has one large restriction that the original sources must be statistically independent. This is the only assumption that we need to take into account in general.

After getting ICs it is necessary to determine the order of the independent components in order to identify normal ECG, noise and abrupt alterations. As the ICs corresponding to noise and abrupt alterations have more distinctive properties than that of original signal both in time and frequency domains, we may employ the statistical properties of these waveforms to recognize the original ECG automatically instead of identifying visually. A random variable for which the PDF is Gaussian distribution can be characterized sufficiently with the mean and variance. If the PDF of variable is non-Gaussian, then the mean and variance are not sufficient as there will be many different constituent signals with the same mean and variance. In ICA one more moment known as the kurtosis is defined in addition to the first two well known moments of random variables, the mean and the variance. The important metrics of ICA are discussed below

The mean (first central moment) of a random variable ' $\boldsymbol{X}$ ' (samples) is defined as;

$$
m_{X}=E[X]=\frac{1}{N} \sum_{i=0}^{N-1} x_{i}
$$

The variance (second central moment), of a random variable ' $\boldsymbol{X}$ ' (samples) is defined as;

$$
\sigma_{X}^{2}=E\left[\left(X-m_{X}\right)^{2}\right]=\frac{1}{N} \sum_{i=0}^{N-1}\left(x_{i}-m_{X}\right)^{2}
$$

The kurtosis is the fourth moment (cumulant) of a random distribution and defined as;

$$
\operatorname{Kurt}(X)=\frac{E\left[\left(X-m_{X}\right)^{4}\right]}{\sigma^{4}}
$$




$$
\operatorname{Kurt}(X)=E\left(X^{4}\right)-3\left[E\left(X^{2}\right)\right]^{2}
$$

The kurtosis is used to measure the non-Gaussianity of a PDF by specifying the relative peaking or flatness of a distribution with respect to a Gaussian distribution.

\subsection{QRS Detection}

The accurate ST segmentation requires an accurate QRS complex detection. An efficient QRS detection method using Hilbert Transform was employed to identify the fiducial point precisely in each heartbeat [16], [17]. The QRS detection procedure is described with help the block diagram as shown in the Fig-4.

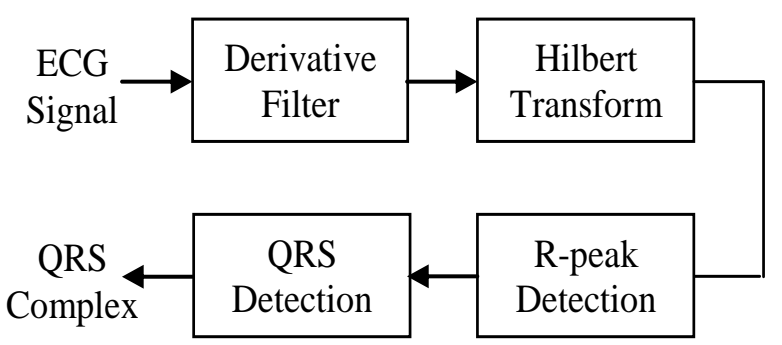

Fig-4 Block diagram of QRS detector

The QRS detection method used in this paper is a naval combination of derivative filter and Hilbert Transform. After the Hilbert Transform, R-peak is detected by setting a threshold to envelope of the transformed and original signals. Later QRS complex is recognized using the slope property of the ECG signal. The first order derivative filter is represented by;

$$
s_{d}(t)=\frac{1}{2 T_{s}}[s(n+1)-s(n-1)]
$$

where $\mathrm{n}=2,3, \ldots, \mathrm{M}-1, \mathrm{M}$ is the total number of samples and $\boldsymbol{T}_{\boldsymbol{s}}$ is the sampling time.

Hilbert Transform has an interesting property that the Transformed version of the signal at the zero crossing of the signal exhibits peaks. Once the R-peak is determined accurately, the other peaks of QRS complex can easily identified with reference to the R-peak using slopes of the ECG signal. To get a signal with zero crossings at peaks of original signal, first the original signal must be differentiated with respect to time. Then the zero crossings of the differentiated ECG signal will be located at the peak positions of the ECG signal. From the Hilbert Transform of the differentiated ECG signal the locations of R-peaks are identified. Hilbert Transform is a simple, efficient and accurate method of detecting QRS complex through detection of R-peaks of ECG waves. The Hilbert transform of real time function, $\mathrm{s}(\mathrm{t})$ is defined as

$$
\hat{s}(t)=H\left[(s(t)]=\frac{1}{\pi} \int_{-\infty}^{\infty} \frac{s(\tau)}{t-\tau} d \tau\right.
$$

The Hilbert transform can also be written as a convolution between $1 / \pi \mathrm{t}$ and $s(\boldsymbol{t})$ as;

$$
\hat{s}(t)=H[s(t)]=\frac{1}{\pi t} * s(t)
$$

The spectrum of the Hilbert transform provides a very useful environment to analyze the signals is given by;

$$
\begin{aligned}
& F[\hat{s}(t)]=\frac{1}{\pi} F\left[\frac{1}{t}\right] F[s(t)] \\
& =\frac{1}{\pi}\left[\int_{-\infty}^{\infty} \frac{1}{s} e^{-j 2 \pi f^{*} s} d s\right] F[s(t)] \\
& F[\hat{s}(t)]=S_{H}(f)=-j \operatorname{sgn}(f) F[s(t)] \\
& =-j \operatorname{sgn}(f) S(f)
\end{aligned}
$$

From (13) it is clear that the Hilbert transform of $s(t)$ is obtained by filtering it through a linear filter with the transfer function, $-j[\operatorname{sgn}(f)]$. Therefore the Hilbert transform is equal to $-\mathrm{j}$ for positive frequency and $+\mathrm{j}$ for negative frequency. Hence it is equivalent to a filter, in which the every frequency component is allowed to pass without any attenuation but their phases are altered by $-\pi / 2$ radians or + $\pi / 2$ radians depending upon the sign of the frequencies. Finally the Fourier transform of the Hilbert transform of $s(t)$ is given by

$$
S_{H}(f)=\mp j F[s(t)]=\mp j S(f)
$$

The analytic signal $z(t)$ can be formed from its Hilbert transform as;

$$
z(t)=s(t)+j s_{H}(t)
$$

The envelope of this analytical signal, $|z(t)|$ is given by;

$$
|z(t)|=\sqrt{s^{2}(t)+s_{H}^{2}(t)}
$$

Subsequently the polar form of the analytical signal is expressed as;

$$
z(t)=B(t) e^{j \phi(t)}
$$

By comparing equations (16) and (17) the envelope, $\boldsymbol{B}(\boldsymbol{t})$ and phase of $z(t)$ can be expressed as;

$$
\begin{aligned}
& B(t)=\sqrt{s^{2}(t)+s_{H}^{2}(t)} \\
& \text { and } \\
& \phi(t)=\tan ^{-1}\left(\frac{s_{H}(t)}{s(t)}\right)
\end{aligned}
$$

The derivative of the $\phi(t)$ is

$$
\omega(t)=\frac{d[\phi(t)]}{d t}=\frac{\frac{d\left[s_{H}(t)\right]}{d t} s(t)-s_{H}(t) \frac{d[s(t)]}{d t}}{B^{2}(t)}
$$

Since the envelope $\mathbf{B}(\mathbf{t})$ coincides with $s(t)$ when $s_{H}(t)=0$ the maximum contribution of the envelope $\mathbf{B}(\mathbf{t})$ is obtained when $s(t)=0$. Thus the Hilbert Transform converts all zero crossing points of the signal into dominant peaks. 


\subsection{STCF feature Extraction}

The ST segment is the wave segment between the J-point and K-points in a time series of ECG wave [18]. First ST segment is extracted from the identified J-point and conventionally located K-point either at $40 \mathrm{~ms}$ (in case of tachycardia) or at $80 \mathrm{~ms}$ after J-point (in case of bradycardia). In this paper a simple algorithm is used to extract ST segments and for the identification of isoelectric line [15]. With forward search from S-peak within a window of $100 \mathrm{~ms} \mathrm{~J}$-point was recognized as one where the ECG signal slope is either zero or changes its sign. With backward search within window of $20 \mathrm{~ms}$ from Q-peak the onset of $\mathrm{Q}$ wave and offset of $\mathrm{P}$ wave are recognized. From the onset of $\mathrm{Q}$ wave and offset of $\mathrm{P}$ wave the PQ-segment is identified and the mean amplitude of PQ-segment is considered as isoelectric line reference. Once the J-point and K-point are known the ST-Segments are extracted by taking the time series of the ECG signal between J-point and $\mathrm{K}$-points in each beat and formed into ST segment feature set [19]. Later the Complexity Factors of each ST segment is computed using the equation (21) and populated into a feature set of ST segment Complexity Factors (STCF) [20], [21]. The Complexity Factor [22] of ST segment is defined as;

$$
\mathrm{CF}=\sqrt{\frac{\sigma_{x "}^{2}}{\sigma_{x}^{2}}}
$$

Where $\sigma_{x}^{2}$, is the variance of second order derivative ST segment and $\sigma_{x}^{2}$ is the variance of the ST segment alone. Simultaneously with STCFs the Instantaneous Heart Rate is also computed from the QRS complexes by using (22) for each beat. These two feature sets, IHRs and STCFs are used for designing and testing the ICA-STCFR model.

$$
\text { Instantaneous heart rate }(I H R)=\frac{60}{\text { RRinterval }} \mathrm{bpm}
$$

\subsection{Linear Regression analysis}

In Linear Regression Analysis (LRA) a linear equation is fitted between two sets of data [23]. To establish a linear regression between two sets of dada the association between them must be strong enough. Normally a scatter-plot is used to assess the strength of the association between any two sets of data. Either increasing or decreasing trends in scatterplot represent some significant relation between the two sets data [24]. If $\boldsymbol{X}$ and $\boldsymbol{Y}$ are two vectors then the values of ' $\boldsymbol{a}$ ' and ' $\boldsymbol{b}$ ' are to be found such that they satisfy the linear equation $\boldsymbol{Y}_{\mathrm{i}}^{\prime}=\boldsymbol{a}+\boldsymbol{b} \boldsymbol{X}_{\mathrm{i}}$. The Linear Regression results in modeling confidents ' $\boldsymbol{a}$ ' and ' $\boldsymbol{b}$ ' as;

$$
\begin{aligned}
& b=\frac{\sum\left(X_{i}-\bar{X}\right)\left(Y_{i}-\bar{Y}\right)}{\sum\left(X_{i}-\bar{X}\right)^{2}} \\
& a=(\bar{Y}-b \bar{X})
\end{aligned}
$$

Where $\bar{X}$ and $\bar{Y}$ are the mean values of feature sets. With the modeling coefficients a regression line is designed using the equation $\boldsymbol{Y}_{\boldsymbol{i}}=\boldsymbol{a}+\boldsymbol{b} \boldsymbol{X}_{\boldsymbol{i}}$ and used as reference threshold for detecting Ischemic beats. Finally the detected beats and the STCF values of all the beats are used for performing ST variability analysis with help of evaluation metrics like Ischemic Intensity Factor (IIF), Ischemic Activity Factor (IAF) and Peak to Average Value (PAV) of Complexity Factors (CFs).

\section{RESULTS}

The ECG datasets acquired from a local hospital was used for designing regression model and testing the proposed method. The ECG data from the first four Leads are preprocessed separately with a bandpass filter to remove baselinewanders, motion artifacts and powerline interference.

For the purpose of illustration the original ECG signals from first four Leads corresponding to the patient ID: 078 and the extracted ECG signal after preprocessing and Independent Component Analysis are shown in the Fig-5 and Fig-6 respectively for few cycles.

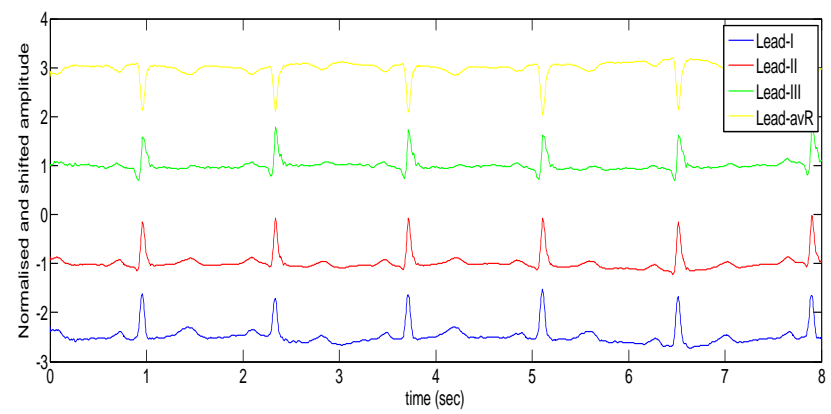

Fig-5: Four Leads of original ECG of the patient ID: 078

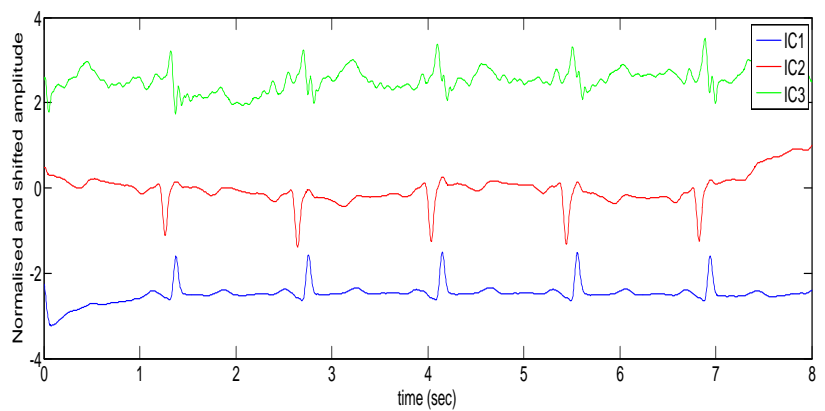

Fig-6: ICs of the ECG Leads of the patient ID: 078

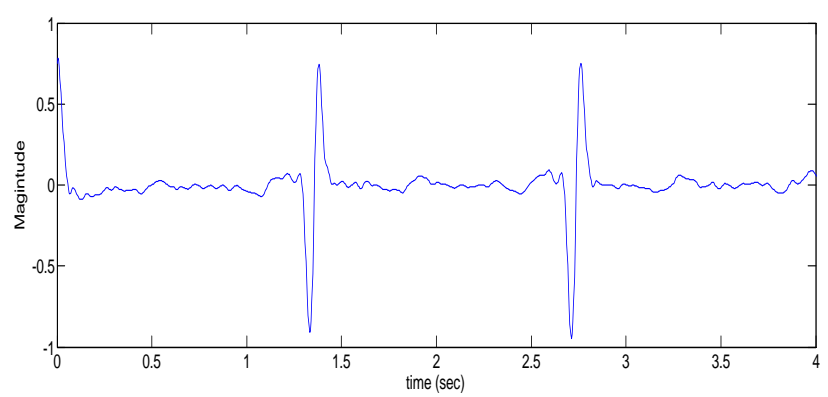

Fig-7(a): ECG after Differentiation 


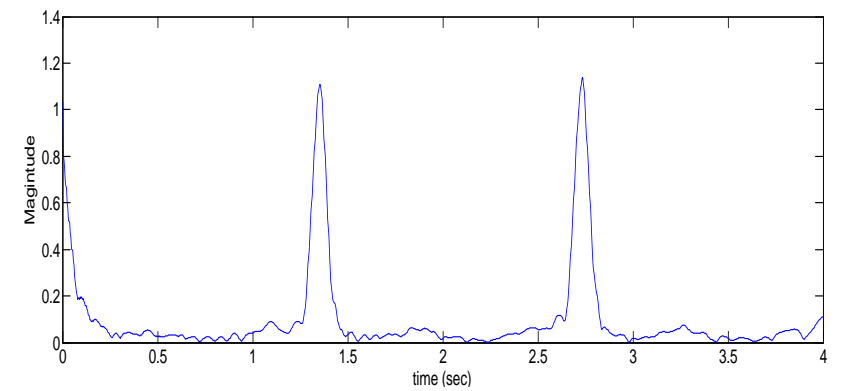

Fig-7(b): ECG after Hilbert Transformation

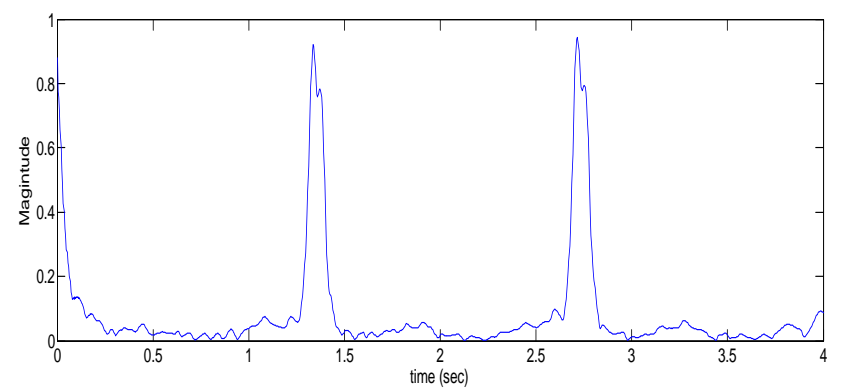

Fig-7(c): Envelope of ECG after Hilbert Transform

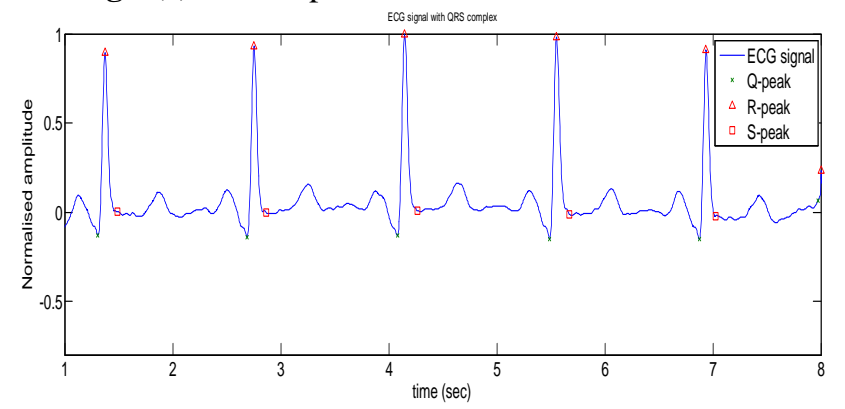

Fig-7(d): QRS of the extracted ECG of the patient ID: 078

The ECG, after extracting from Independent Component Analysis, is processed with QRS detection algorithm using Hilbert Transform. This algorithm involves differentiation, Hilbert Transform (HT), Envelop detection and QRS Complex detection. For instance the results of series of operations in QRS detection using HT are illustrated in the Fig-7. Next the ST segment of each beat is extracted from the extracted ECG. Later the ST segments Complexity Factors (STCFs) of each beat are computed using the mean amplitude of the PQ-segment as the isoelectric reference level. From the RR intervals of each beat cycle the Instantaneous Heart Rate (IHR) is computed using (12) for each beat.

$$
\text { Instantaneous heart rate }(I H R)=60 / R R \text { bpm }
$$

Both STCF levels and Instantaneous heart rates (IHRs) are used as feature sets for the ST analysis. For instance the IHR and STCF levels of ECG signal of the patient ID: 078 for first 30 beats are tabulated in the Table-1. A threshold is derived from the Linear Regression model that is designed between IHR and STCF levels from the Table-1 to derive design coefficients. Theses model coefficients are used to set a threshold for beat classification. The regression model threshold is set from the modeling coefficients as visualized in the Fig-8. This regression line is used as a reference threshold to compare the STCFs of the testing feature sets
Table-1: IHRs and STCFs of the ECG of patient ID: 078

\begin{tabular}{|c|c|c|c|c|c|}
\hline $\begin{array}{c}\text { Beat } \\
\text { No }\end{array}$ & $\begin{array}{c}\text { IHR } \\
\text { (bpm) }\end{array}$ & STCF & $\begin{array}{c}\text { Beat } \\
\text { No }\end{array}$ & $\begin{array}{c}\text { IHR } \\
\text { (bpm) }\end{array}$ & STCF \\
\hline 1 & 54 & 0.0090 & 16 & 78 & 0.2575 \\
\hline 2 & 45 & 0.0100 & 17 & 77 & 0.0636 \\
\hline 3 & 79 & 0.1649 & 18 & 77 & 0.1163 \\
\hline 4 & 78 & 0.2575 & 19 & 78 & 0.0686 \\
\hline 5 & 77 & 0.0636 & 20 & 102 & 0.0352 \\
\hline 6 & 77 & 0.1163 & 21 & 79 & 0.1649 \\
\hline 7 & 78 & 0.0686 & 22 & 78 & 0.2575 \\
\hline 8 & 102 & 0.0352 & 23 & 77 & 0.0636 \\
\hline 9 & 79 & 0.1649 & 24 & 77 & 0.1163 \\
\hline 10 & 78 & 0.2575 & 25 & 78 & 0.0686 \\
\hline 11 & 77 & 0.0636 & 26 & 102 & 0.0352 \\
\hline 12 & 77 & 0.1163 & 27 & 79 & 0.1649 \\
\hline 13 & 78 & 0.0686 & 28 & 78 & 0.2575 \\
\hline 14 & 102 & 0.0352 & 29 & 77 & 0.0636 \\
\hline 15 & 79 & 0.1649 & 30 & 77 & 0.1163 \\
\hline
\end{tabular}

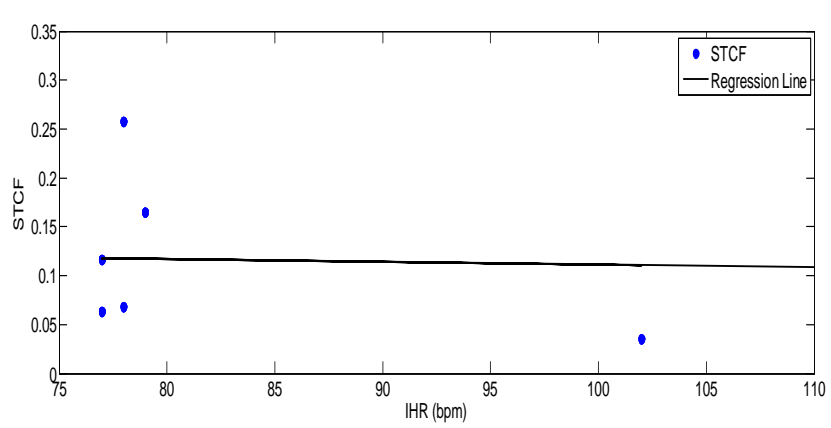

Fig-8: Linear Regression between STCFs with IHRs

and the decision is made in favorer of probable Ischemic beats if the value of STCF of the testing feature set is more than that obtained from the regression model. Later with a hard threshold final and true Ischemic beats are separated from the probable Ischemic beats. The separated Ischemic beats are populated for further ST variability analysis. Using (13), (14) and (15) the parameters, Ischemic Intensity Factor, Ischemic Activity Factor (per 30 beats) and Peak to Average Value (PAV) respectively are evaluated and the ST variability is analyzed.

$$
\begin{gathered}
\text { Ischemic Intensity Factor }(I A F)=\frac{N_{I s c}}{N_{T}} \\
\text { Ischemic ActivityFactor }(I A F)=N_{I s c} * C F_{a v g} \\
\text { Peakto AverageValue }(P A V)=\frac{C F_{\text {peak }}}{C F_{\text {avg }}}
\end{gathered}
$$

Where $N_{I s c}$ is the number of detected Ischemic beats, $N_{T}$ is the total number of beats, $\boldsymbol{C F}_{\text {avg }}$ is the average STCF level and $\boldsymbol{C F}_{\text {peak }}$ is the peak value of the respective ECG signal ST segments. 
Table -2: Performance Evaluation and Comparison

\begin{tabular}{|c|c|c|c|c|c|c|c|}
\hline \multicolumn{2}{|c|}{ Patient ID } & 57 & 77 & 78 & 1076 & 1077 & 1115 \\
\hline \multirow{4}{*}{ 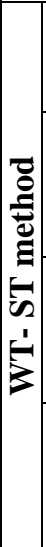 } & $\begin{array}{c}\text { No of } \\
\text { Ischemic } \\
\text { beats }\end{array}$ & 2 & 2 & 3 & 3 & 5 & 7 \\
\hline & $\begin{array}{c}\text { Ischemic } \\
\text { Intensity } \\
\text { Factor } \\
\text { (IIF) }\end{array}$ & 0.067 & 0.067 & 0.100 & 0.100 & 0.167 & 0.233 \\
\hline & $\begin{array}{c}\text { Ischemic } \\
\text { Activity } \\
\text { Factor } \\
\text { (IAF) }\end{array}$ & 0.003 & 0.001 & 0.004 & 0.003 & 0.055 & 0.016 \\
\hline & $\begin{array}{c}\text { Peak to } \\
\text { Average } \\
\text { Value } \\
\text { (PAV) }\end{array}$ & 5.18 & 9.24 & 5.46 & 6.40 & 2.569 & 6.47 \\
\hline \multirow{4}{*}{ 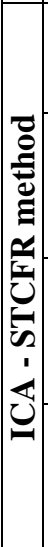 } & $\begin{array}{c}\text { No of } \\
\text { Ischemic } \\
\text { beats }\end{array}$ & 12 & 10 & 5 & 9 & 11 & 9 \\
\hline & $\begin{array}{l}\text { Ischemic } \\
\text { Intensity } \\
\text { Factor } \\
\text { (IIF) }\end{array}$ & 0.400 & 0.333 & 0.167 & 0.300 & 0.367 & 0.300 \\
\hline & $\begin{array}{l}\text { Ischemic } \\
\text { Activity } \\
\text { Factor } \\
\text { (IAF) }\end{array}$ & 0.084 & 0.065 & 0.019 & 0.039 & 0.043 & 0.024 \\
\hline & $\begin{array}{l}\text { Peak to } \\
\text { Average } \\
\text { Value } \\
\text { (PAV) }\end{array}$ & 1.54 & 1.97 & 2.24 & 2.49 & 2.64 & 1.55 \\
\hline
\end{tabular}

For the patient ID: 078 the parameters the IIF, IAF and PAV are evaluated as $0.167,0.019$ and 2.24 respectively for ICASTCFR method. Similarly these parameters are evaluated for all the six patients' ECG data using both WT-ST method and ICA-STCFR methods and tabulated the results in the Table-2.

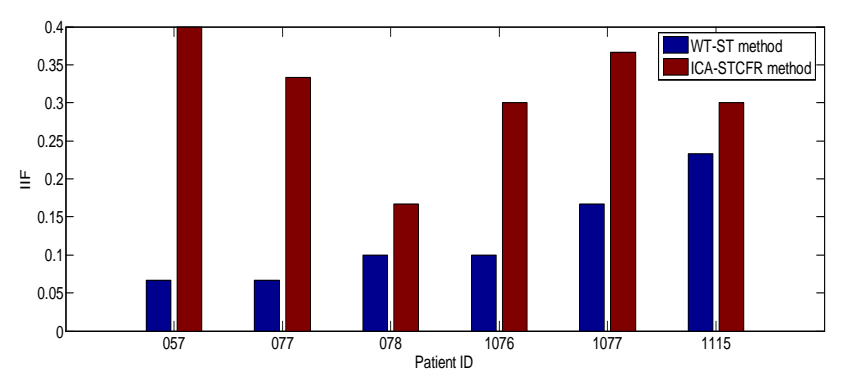

Fig-9: Patient wise Ischemic Intensity Factor

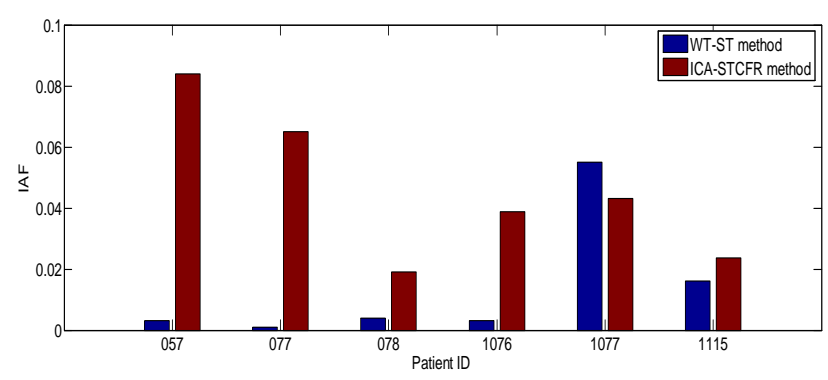

Fig-10: Patient wise Ischemic Activity Factor
Fig-9 and Fig-10 shows the patient wise IIF and IAF respectively for all six patients according the Table-2 data. From the Table-2 and the Fig-9 and Fig-10 it is obvious that the ICA-STCFR (Independent Component Analysis - ST segment Complexity Factor Regression modeling) method of ST variability analysis is yielding higher Ischemic Intensity Factor (IIF), Ischemic Activity Factor (IAF) and Peak to Average Values (PAV) than the Wavelet Transform (WT-ST) based method.

\section{CONCLUSIONS}

A new method based on ST segment Complexity Factor (STCF) feature, Independent Component Analysis, Hilbert Transform and Linear Regression is proposed in this paper. The Complexity Factor is an effective and suitable feature for detection of myocardial ischemia. The Independent Component Analysis greatly reduces the effect of noise present in the passband of the ECG and increases the Signal to Noise Ratio than other methods as the ECG is extracted by reducing the multi-dimensionality of ECG leads through independence. The Complexity Factor improves the accuracy of Ischemic detection by taking the variance of the ST segment into account rather than mean of ST levels as in WT-ST method. The regression threshold decreases the probability of missing Ischemic beats and increases the classification accuracy. The performance of the model was evaluated with Ischemic Intensity Factor (IIF), Ischemic Activity Factor (IAF) and Peak to Average Value (PAV) by employing the designed model on the ECG signals acquired from a local hospital. The proposed method is giving better results compared to the WT-ST method for all the patients. Thus the ICA-STCFR modeling for analysis of ST variability will support the doctors significantly to recognize the manifestation of Ischemia and diagnose the cardiac diseases accurately.

\section{REFERENCES}

[1]. Coumel P. and Garfein O, "Electrocardiography: Past and Future", Annuals of the New York, Academy of Sciences, Vol. 601, New York, 1990.

[2]. J.R. Hampton, "The ECG Made Easy", International Edition (English), $8^{\text {th }}$ Edition, Elsevier Health Sciences, 2013.

[3]. N. Goldschlager, "Principles of Clinical electrocardiography", $13^{\text {th }}$ edition, ISBN 978-083-8579-510, Connecticut, USA.

[4]. G.-Y. Jeong, K.-H Yu, "Design of Ambulatory ECG Monitoring System to detect ST pattern change," SICEICASE International Joint Conference, Oct 2006.

[5]. Li, C., Zheng, C., Tai, C., "Detection of ECG characteristic points using wavelet transforms", IEEE Trans. Biomed. Eng. 1995; 42:21-28.

[6]. J. Pan and W. J. Tompkins, "A real-time QRS detection algorithm", IEEE Trans. Biomed. Eng., vol. 32, pp. 230-236, March 1985.

[7]. P. S. Hamilton, W. J. Tompkins, "Quantitative Investigation of QRS Detection Rules Using MIT/BIH Arrhythmia Database", IEEE Transactions on 
Biomedical Engineering, Vol.31, No.3, pp. 1157-1165, ISSN 0018-9294, March 2007.

[8]. Dr. Christian, “A DSP Practical Application: Working on ECG Signal, Applications of Digital Signal Processing", Cuadrado-Laborde (Ed.), ISBN: 978-953307-406-1.

[9]. Kaur, M., Singh, B., Ubhi, JS and Seema Rani, "Digital Filtration of ECG Signals for Removal of Baseline Drift", International Conference on Telecommunication Technology and Applications Proc of CSIT vol.5, pp: no.105-109, 2011

[10]. Zarzoso V, Comon P, "Robust independent component analysis for blind source separation and extraction with application in electrocardiography", In: Proc. EMBC2008, 30th Annual International Conference of the IEEE Engineering in Medicine and Biology Society, Vancouver, BC, Canada, 3344-3347, 2008.

[11]. P. S. Hamilton, "A comparison of adaptive and nonadaptive filters for the reduction of powerline interference in the ECG", IEEE Trans. Biomed. Eng, 1996; 43:105-109.

[12]. M.P.S. Chawla, "Detection of indeterminacies in corrected ECG signals using parameterized multidimensional independent component analysis", Computational and Mathematical Methods in Medicine Vol. 10, No. 2, 85-115, June 2009.

[13]. J. F. Cardoso, "Blind signal separation: Statistical principles," Proc. IEEE, vol. 86, pp. 2009-2025, Oct. 1998.

[14]. P. Comon, "Independent component analysis, a new concept?," Signal Processing, vol. 36, no. 3, pp. 287314, 1994.

[15]. K. Abed Meraim, M.G. Amin, and A.M. Zoubir, "Joint anti-diagonalization for blind source separation", Proc. ICASSP-01, 2001.

[16]. D. Benitez, P. Gaydecki, A. Zaidi, and A. Fitzpatrick, "A new QRS detection algorithm based on the Hilbert transform," in Computers in Cardiology 2000, 2000, pp. 379-382.

[17]. S. L. Hahn, "Hilbert transforms," in The Transforms and Applications Handbook (A. Poularakis, Ed.), Boca Raton FL: CRC Press, 1996, ch. 7.

[18]. X. Li, et al., "ST-T complex automatic analysis of the electrocardiogram signal based on wavelet transform," Proc. 29th IEEE Bioengineering, pp. 144-145, 2003.

[19]. Lehtinen, R., "ST/HR Hysteresis: Exercise and Recovery Phase ST Depression/Heart Rate Analysis of the Exercise ECG," J. Electrocardiol., No. 32, 1999, pp. 198-204.

[20]. Hjorth, B., "The physical significance of time domain descriptors in EEG analysis", Electroenceph. ClinNeurophysiol.1973, 34:321-325. 4.

[21]. Hjorth, B., "EEG analysis based on time domain properties", Electroencephalography and Clinical Neurophysiology, vol. 29, no.3, pp. 306-310, September-1970.

[22]. Hjorth, B., "Time domain descriptors and their relation to a particular model for generation of EEG activity", In: G. Dolce, H. Künkel (Eds.) CEAN:
Computerized EEG Analysis. Gustav Fischer, Stuttgart; 1975:3-8.

[23]. Barlow, Jesse L., "Numerical aspects of Solving Linear Least Squares Problems", In Rao, C.R. Computational Statistics, Handbook of Statistics, 9, North Holland. ISBN 0-444-88096-8:1993.

[24]. Edwards, A. L., "An Introduction to Linear Regression and Correlation", San Francisco, CA: W. H. Freeman, pp. 20-32, 1976

\section{BIOGRAPHIES}

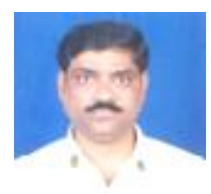

S. Thulasi Prasad received B.Tech degree from Sri Venkateswara University, Tirupati, Andhra Pradesh, India in Electronics and Communication Engineering in 1996, M.Tech degree in Electronics and Communication Engineering from Sri Venkateswara University, Tirupati, Andhra Pradesh, India in 2003. He has 17 years of teaching experience and 2 years of industrial experience. Presently he is working as Associate Professor in the Dept. of ECE in Sree Vidyanikethan Engineering College, Tirupati, Andhra Pradesh, India

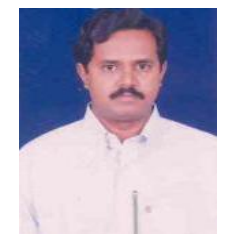

Dr. S. Varadarajan received B.Tech degree from Sri Venkateswara University, Tirupati, Andhra Pradesh, India, in Electronics and Communication Engineering in 1987, M.Tech degree from Regional Engineering College, Warangal, Andhra Pradesh, India in 1993 and Ph.D from Sri Venkateswara University, Tirupati, Andhra Pradesh, India in 2005. Presently he is working as Professor in the Department of Electronics and Communication Engineering, Sri Venkateswara University College of Engineering, Tirupati. His research areas are Signal and Image Processing, Radar Signal Processing and Digital Communications. He is a member of IETE, ISTE and IEEE. $\mathrm{He}$ is the chairperson of IETE Tirupati centre. He has teaching experience of 23 years. 